\title{
Avaliação respiratória de crianças com e sem diagnóstico de asma
}

Respiratory evaluation of children

with and without asthma diagnosis

FisiSenectus. Unochapecó Ano 1, n. 2 - Jul./Dez. 2013 p. $47-55$

\section{Indiamara de Oliveira Flores Dal Magro Silvani}

Fisioterapeuta e Mestre em Engenharia Biomédica, professora do curso de Fisioterapia na Unochapecó.

\section{Aline Galli}

Fisioterapeuta, graduada pela Universidade Comunitária da Região de Chapecó (Unochapecó).

\section{Talita Lauschner Castelli}

Fisioterapeuta, graduada pela Universidade Comunitária da Região de Chapecó (Unochapecó).

\section{Resumo}

Introdução: a asma brônquica é uma doença inflamatória crônica das vias aéreas que se caracteriza por obstrução reversível espontaneamente ou com tratamento. A maior prevalência é entre crianças, configurando assim um sério problema de saúde. Esse estudo teve como objetivos identificar quais as diferenças entre a forma do tórax, avaliar o padrão respiratório, avaliar a pressão expiratória e inspiratória máxima e ainda o pico de fluxo expiratório. Material e métodos: a presente pesquisa caracteriza-se pelo método quantitativo, tendo uma amostra de 40 crianças entre 06 a 12 anos de idade, sendo 20 sem diagnóstico de asma brônquica (Grupo 1), e 20 com diagnóstico de asma brônquica (Grupo 2). Para a análise dos resultados foi utilizado a média, desvio padrão e Teste $t$ de Student, apresentados através de tabelas descritivas. Resultados e discussão: podese concluir que em algumas medidas e análises o grupo de crianças asmáticas atingiu resultados inferiores em relação ao grupo de crianças saudáveis. O asmático em função das características da doença apresenta um aumento da resistência ao fluxo aéreo, aprisionamento de ar e hiperinsuflação pulmonar, o que levam a alterações mecânicas na dinâmica tóraco-abdominal e à desvantagem muscular. Conclusão: a avaliação adequada da mecânica respiratória, juntamente com tratamento da asma na infância são importantes fatores para a prevenção secundária de redução da função pulmonar e de doença pulmonar crônica na vida adulta. São necessários mais estudos e com uma amostra maior para que seja possível estabelecer um consenso a esse respeito, principalmente estudos com crianças, em que verificamos que o número de pesquisas com este público é menor, a fim de produzir maiores conhecimentos a este assunto.

\section{Palavras-chave}

Asma. Fisioterapia. Sistema respiratório.

\section{Fisiß̌enectus}




\begin{abstract}
Introduction: bronchial asthma is an inflammatory disease of the airways characterized by the reversible obstruction spontaneously or with treatment. The highest prevalence is among children, constituting a serious health problem. This study aimed at identifying the differences between the thorax shapes, evaluating the breathing pattern and also aimed at estimating the maximum inspiratory and expiratory pressure, as well as the peak expiratory flow. Material and methods: this research is characterized by a quantitative method, having a sample of 40 children from 06 to 12 years old, being 20 without diagnosis of bronchial asthma (Group 1), and 20 diagnosed with bronchial asthma (Group 2). The mean, the standard deviation and Test $t$ of Student were used to analyze the results, shown through descriptive tables. Results and discussion: in conclusion, in some measurements and analyses, the asthmatic children group presented inferior results when compared to the healthy children group. The asthmatic group, because of the disease characteristics, presents an increase of resistance to the air flow, air trapping and pulmonary hyper-insufflations, which takes to mechanical alterations in the thoracic-abdominal dynamic and muscular disadvantage. Conclusion: the adequate evaluation of the respiratory mechanic, along with asthma treatment in childhood are important factors to secondary prevention of the pulmonary function reduction and the acute pulmonary disease in adult age. More studies and a bigger sample are necessary to enable a consensus about this, mainly studies with children, in order to develop more knowledge on this issue; since it was verified that the number of research with this public is smaller.
\end{abstract}

\title{
Keywords
}

Asthma. Physical therapy specialty. Respiratory system.

\section{Introdução}

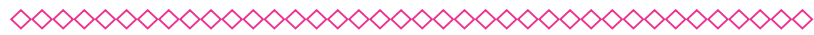

A prevalência da asma vem aumentando nas últimas décadas, sendo considerada, atualmente, uma das principais doenças crônicas no mundo. $\mathrm{Na}$ infância constitui uma importante causa de morbidade, resultando em comprometimento de atividades diárias e faltas escolares, relacionada à necessidade de cuidados em serviços de emergência médica, bem como a hospitalizações frequentes ${ }^{1}$.

A asma é uma patologia de diagnóstico complicado e pode ser confundida com outras patologias respiratórias, assim como, a asma no adulto e na criança possui algumas particularidades. A asma e seus sintomas respiratórios são muito comuns na infância, e vários estudos têm demonstrado sua associação com mecânica respiratória e função pulmonar prejudicada na vida adulta. Além disso, alguns autores demonstraram que mecânica respiratória e função pulmonar prejudicada na infância é um fator de risco para função pulmonar anormal na vida adulta, concluindo que o prognóstico da asma ou dos sintomas respiratórios na infância é desfavorável ${ }^{1,2}$.

A asma é, atualmente, definida como doença inflamatória crônica das vias aéreas que, em indiví- duos susceptíveis, causa episódios recorrentes de sibilos, dificuldade respiratória e tosse, principalmente à noite e no início da manhã. Os episódios são, usualmente, associados à obstrução variável e difusa do fluxo aéreo, geralmente reversível parcial ou total, espontaneamente ou após tratamento. A inflamação também aumenta a reatividade brônquica a vários estímulos inespecíficos. Resulta de uma interação entre genética, exposição ambiental e outros fatores específicos que levam ao desenvolvimento e à manutenção dos sintomas ${ }^{3,4,5}$.

A maior prevalência da asma é entre crianças, configurando, assim, um sério problema de saúde. Cerca de $80 \%$ das crianças asmáticas desenvolvem os primeiros sintomas antes dos cinco anos de idade. Apesar dos avanços sobre o conhecimento da fisiopatologia e da terapêutica específica, a gravidade da asma e a prevalência parecem estar aumentando cada vez mais ${ }^{6}$.

Na infância, a asma apresenta um quadro clínico de características variadas, as quais geralmente são difíceis de diferenciar em relação a outras causas de obstrução das vias aéreas inferiores. A maioria das crianças com asma apresenta episódios recorrentes de sibilância, tosse e/ou dispneia, os quais são geralmente desencadeados por infecções virais ou ainda por exposição a fatores am- 
bientais, tais como alérgenos, ar frio, fumaça de cigarros etc. $^{7}$

O asmático, em função das características da doença, experimenta aumento da resistência ao fluxo aéreo, aprisionamento de ar e hiperinsuflação pulmonar, o que levam a alterações mecânicas na dinâmica tóraco-abdominal e à desvantagem muscular. 0 remodelamento das vias aéreas constitui a principal causa de perda de reversibilidade da obstrução ao fluxo aéreo em asmáticos. A hiperinsuflação dos pulmões aplaina o diafragma e limita os músculos inspiratórios, levando-os a desvantagem mecânica. A fraqueza dos músculos respiratórios eleva a morbidade e a mortalidade dessa doença. Sendo assim, a medida da força muscular respiratória, por meio da quantificação das pressões respiratórias (inspiratória/expiratória) máximas, deve ser incluída na avaliação do asmático ${ }^{8}$

Quanto à gravidade, a asma (fora da crise) pode ser intermitente, persistente leve, persistente moderada e persistente grave, tendo como parâmetros para a classificação frequência dos sintomas (dispneia, aperto no peito, sibilos e tosse), grau de comprometimento das atividades diárias, número e intensidade das crises e medicação necessária para estabilização dos sintomas, necessidade de consultas de emergências e hospitalização, frequência de sintomas noturnos, frequência do uso de broncodilatadores para alívio dos sintomas e valor do Pico de Fluxo Expiratório (PFE) 9 .

A avaliação respiratória em asmáticos é de suma importância tanto no diagnóstico da asma quanto na avaliação do tratamento, sendo a manutenção da função pulmonar normal um dos objetivos a serem alcançados. Dentre os itens utilizados para avaliação, a pressão inspiratória máxima e pressão expiratória máxima e o Pico de Fluxo Expiratório são considerados importantes, geradas por meio da utilização de parelhos específicos para a realização dessas medidas respiratórios e por serem métodos simples e não invasivos ${ }^{8}$.

Apesar do reconhecimento da importância e da utilidade na avaliação respiratória no manejo da asma, poucos são os estudos nacionais que descrevem as características da força muscular e do PFE de crianças e adolescentes asmáticas, assim como suas particularidades decorrentes de variáveis, como a gravidade e o tempo de manifestação da doença.

Ainda dentro da avaliação, um quesito muito importante é o exame físico, observando a morfologia do tórax com suas possíveis distorções, expansibilidade e mobilidade torácica, o padrão ventilatório com vista na ação diafragmática e ainda o acompanhamento dos sinais vitais (frequência cardíaca, frequência respiratória, pressão arterial e saturação de $\mathrm{O}_{2}$ ) no repouso e na execução de atividade física.

O tratamento do asmático, em especial da criança, tem como objetivo prevenir a mortalidade, reduzir a morbidade expressa pela hospitalização e atendimentos de urgência, manter o paciente livre de sintomas ou crises, manter a função pulmonar normal ou próxima do normal e propiciar um crescimento normal das crianças, livres de efeitos colaterais.

As avaliações adequadas da mecânica respiratória, juntamente com tratamento da asma na infância, são importantes fatores para a prevenção secundária de redução da função pulmonar e de doença pulmonar crônica na vida adulta. Com isto, faz-se necessário informações sobre o comprometimento da musculatura respiratória na asma; de acordo com os níveis de gravidade, permite planejar estratégias públicas para a assistência.

No presente estudo, foram realizados alguns itens da avaliação respiratória de crianças com e sem diagnóstico de asma para compararmos os resultados encontrados.

\section{Materiais e métodos}

$\infty<\infty<\infty<\infty<\infty<\infty<\infty<\infty<\infty<\infty<\infty<\infty<\infty<\infty<\infty<\infty$

A presente pesquisa caracteriza-se pelo método quantitativo. Em relação à abordagem quantitativa, está relaciona à quantificação de dados obtidos mediante pesquisa. Para o emprego dessa forma, são necessários recursos e técnicas estatísticas, os quais podem variar em termos de complexidade, que vai desde a mais simples, como porcentagem, média, moda, mediana e desvio-padrão, até as de uso mais complexo, como coeficiente de correlação, análise de regressão ${ }^{10}$. 
Aconselha-se a utilização dessa abordagem em procedimentos descritivos, nos quais se procura descobrir e classificar a relação entre variáveis, bem como nas investigações que procuram determinar relações de causalidade entre fenômenos ${ }^{10}$.

A amostra é o subconjunto extraído de uma população, sendo que partes desta são selecionadas segundo critérios que garantam sua representatividade $^{11}$. A amostra foi constituída por quarenta crianças entre 6 a 12 anos de idade. Dentre estas, vinte sem diagnóstico de asma brônquica (Grupo 1) e sem história de doenças respiratórias ou sistêmicas, e vinte crianças que possuem diagnóstico de asma brônquica, sem nenhuma outra doença associada (Grupo 2). A amostra que compõe o Grupo 1 foi obtida nas escolas municipais de São Carlos (SC), enquanto a amostra do Grupo 2 foi obtida no Serviço Municipal de Saúde do mesmo município.

Para a realização desta pesquisa, foi solicitada, primeiramente, a aprovação do Comitê de Ética em Pesquisas da Universidade Comunitária da Região de Chapecó (Unochapecó), a qual foi aprovada com protocolo n. 008/11.

O primeiro contato aconteceu com os secretários municipais da Saúde e da Educação. Esse teve por objetivo apresentar o projeto, sanar dúvidas e obter a declaração de concordância por escrito para que o projeto pudesse ser efetivamente realizado. Após, foi solicitada uma autorização para a Secretaria Municipal de Saúde de São Carlos (SC), para que, por meio das unidades básicas de saúde do município, pudéssemos ter acesso às crianças com diagnóstico clínico de asma que estavam ou estiveram em acompanhamento nas unidades. Solicitamos, ainda, autorização à Secretaria Municipal da Educação para podermos realizar a avaliação de crianças sem o diagnóstico de asma.

Após estes procedimentos iniciais, foi encaminhada a declaração de concordância das instituições, diretamente para as escolas já com a autorização vinda da Secretária Municipal da Educação, para podermos realizar a avaliação dentro do estabelecimento.

Em momento subsequente, entrou-se em contato com os responsáveis pelas crianças, informando-os da realização da pesquisa e solicitando a autorização através dos Termos de Consentimento Livre e Esclarecido. Estando de acordo em participar, e respeitando os critérios de inclusão e exclusão da pesquisa, os responsáveis assinaram o Termo de Consentimento Livre e Esclarecido, o que permitiu o início da pesquisa. 0 termo foi disponibilizado em duas vias, uma para o participante do estudo e outra para as acadêmicas responsáveis pela pesquisa.

De acordo com a Resolução n. 196/96 do Conselho Nacional de Saúde (CNS), o Consentimento Livre e Esclarecido protege os indivíduos participantes do projeto que irá ser realizado. Considerando que a pesquisa envolve seres humanos, deve-se sempre tratá-los em sua dignidade, respeitá-los em sua autonomia e defendê-los em sua vulnerabilidade.

A avaliação se apresenta como uma forma comparativa dos valores da Pressão inspiratória máxima $\left(\mathrm{Pi}_{\max }\right)$, da Pressão expiratória máxima (Pemax), identificar o tipo de tórax, o padrão ventilatório e o pico de fluxo expiratório (PFE) de crianças com e sem diagnóstico de asma.

Para avaliar as crianças, foram coletadas informações do tipo identificação do paciente, diagnóstico clínico, queixa principal, história da doença atual, antecedentes patológicos, hábitos de vida, entre outros quesitos importantes para a avaliação inicial.

Para a avaliação da forma do tórax, este foi observado visualmente e por intermédio de inspeções na região. Os tipos de tórax avaliados foram de pombo, que se caracteriza com o esterno mais proeminente e as costelas horizontalizadas; plano que se caracteriza pela diminuição do diâmetro ântero-posterior; tonel caracterizado por um aumento exagerado do diâmetro ântero-posterior; sapateiro, que se caracteriza por depressão da parte inferior do esterno e região epigástrica; sino, que se caracteriza por ter a parte inferior exageradamente alargada ${ }^{12}$.

Para avaliação do padrão respiratório, foi utilizada a classificação quanto aos padrões existentes, sendo este diafragmático, intercostal e costal superior.

A manovacuometria foi realizada por meio das medidas de $\mathrm{Pi}_{\max }$ e $\mathrm{Pe}_{\text {max' }}$ segundo o método preconizado por Black e Hyatt (1969), por meio de um manuvacuômetro. 
Para avaliação do PFE, foi utilizada a mensuração através do aparelho ASSESS ${ }^{\circledR}$ Full Range Peak Flow Meter (60-880 I/min), da marca Respironics ${ }^{\circledR}$, com bocais descartáveis para todas as crianças, nas quais foram realizadas pelo menos três medidas satisfatórias de cada pico, ou seja, sem vazamento de ar pela boca ou nariz, sendo considerado o maior valor obtido.

Após a coleta de dados, para conseguir descrever o perfil da amostra, através das diversas variáveis encontradas no grupo, foram feitas tabelas estatísticas e descritivas com os valores das médias das idades, tipo de tórax, padrão ventilatório, valores da média e desvio padrão da $\mathrm{Pi}_{\max }$ e $\mathrm{Pe}_{\text {max' }^{\prime}}$ e para o PFE.

Para análise dos dados, foi empregado o Teste t de Student, sendo este um teste paramétrico de largo uso, geralmente adotado quando o pesquisador possui amostras iguais ou inferiores a trinta unidades e as variâncias paramétricas são desconhecidas ${ }^{13}$, e com intervalo de confiança (IC) de $95 \%$, considerando um teste significativo quando o valor de $p$ for igual ou menor que 0,05 . 0 objetivo da utilização deste é analisar a diferença encontrada entre as medidas de $\mathrm{Pi}_{\max }$ e $\mathrm{Pe}_{\text {max }^{\prime}}$ e o PFE entre o Grupo 1 e o Grupo 2. 0 desvio padrão serviu para medir a dispersão dos valores individuais em torno da média dos valores encontrados.

\section{Resultados e discussão}

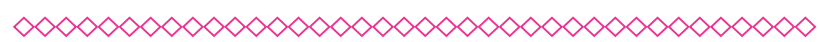

A análise dos resultados obtidos no presente trabalho revela que em relação à amostragem de crianças asmática estudadas, houve uma prevalência do sexo masculino em relação ao feminino, sendo $55 \%$ do sexo masculino e $45 \%$ do grupo do sexo feminino. Que vem confirmar o que Ferrari descreveu em seu estudo, em que relata que até a adolescência há um ligeiro predomínio na doença do sexo masculino ${ }^{14}$.

Estudos mostram que crianças do sexo masculino têm maiores chances de desenvolverem asma, uma vez que suas vias aéreas têm menor diâmetro e maior tônus do que as de crianças da mesma idade e do sexo feminino, o que resulta em menor fluxo pulmonar ${ }^{15}$. Estudo realizado na Inglaterra mostra a prevalência de hospitalização por problemas respiratórios aos sete anos, sendo que no sexo feminino foi de $4,5 \%$ e no sexo masculino $6,9 \%^{16}$. Esses dados são similares aos que foram encontrados no presente estudo, no qual se constatou uma maior porcentagem de prevalência da asma no sexo masculino, na faixa etária de 6 a 12 anos.

Em relação à idade, obteve-se uma média do grupo de crianças asmáticas de 8,3, e do grupo de crianças sem diagnóstico de asma em 8,7 (tabela 1). Foi encontrado no projeto ISAAC (International Study of Asthma and Allergies in (hildhood) que a prevalência da asma é de $5,4 \%$ na idade de 6 a 7 anos e de $15 \%$ entre 13 e 14 anos $^{17}$.

Das vinte crianças asmáticas avaliadas, 11 apresentaram padrão respiratório do tipo costal superior, seis delas do tipo intercostal e três com padrão respiratório diafragmático (tabela 2). $\mathrm{Na}$ literatura se evidencia que o asmático apresenta alteração da mecânica ventilatória com rebaixamento das cúpulas diafragmáticas e redução de seu trajeto durante os movimentos respiratórios, prejudicando a ventilação basal. Dessa forma, a caixa torácica adota uma atitude em inspiração, levando a diminuição da mobilidade costal, caracterizando a respiração torácica superior, em que leva a um grande consumo de energia ${ }^{18}$.

Ao avaliar o tipo de tórax (tabela 3) no grupo das crianças asmáticas, o predominante ficou do tipo sapateiro e plano, enquanto em comparação com o grupo de não asmáticos este ficou com o tipo de tórax plano.

Ao realizar a inspeção de tórax, observamos os movimentos respiratórios, sua frequência, sua regularidade e sua profundidade, bem como os esforços inspiratórios e expiratórios relativos ${ }^{19}$.

É de suma importância na avaliação do tipo de tórax identificar se ocorrem retrações ou abaulamentos, observando assim se existem alterações a nível vertebral, de costelas ou de esterno. Deve-se dar atenção para este quesito da avaliação, pois se apresentar alterações pode limitar a mecânica respiratória.

Ao comparar as amostras do Grupo 1 e Grupo 2 , em relação a força muscular respiratória $\left(\mathrm{Pi}_{\text {max }} \mathrm{e}\right.$ $\left.\mathrm{Pe}_{\max }\right)$, obteve-se resultados diferentes entre eles (tabela 4). 
$\mathrm{A} \mathrm{Pi}_{\max }$ é um índice da força dos músculos inspiratórios (diafragma e intercostais), enquanto a $\mathrm{Pe}_{\max }$ mede a força dos músculos expiratórios (abdominais e intercostais) ${ }^{20}$.

As crianças asmáticas, ao desencadear uma crise, podem apresentar uma obstrução expiratória das vias aéreas de forma agudizada. Isso leva a uma hiperinsuflação e ainda a uma resistência à passagem de ar, dificultando a melhor execução da musculatura respiratória de forma funcional. Ou seja, gera um aumento do trabalho da musculatura inspiratória, que se torna encurtada e, por conseguinte, menos eficiente na geração da força de tensão, representando uma carga adicional a ser vencida $8,21,22$.

Este ciclo pode explicar a perda gradativa da força muscular respiratória, pois evidencia uma diminuição na habilidade contrátil desta musculatura de crianças portadoras de asma brônquica. Frente a isto, percebe-se a relevância da avaliação da força muscular respiratória por meio das quantificações das pressões respiratórias máximas para monitoramento da gravidade da patologia.

Na presente pesquisa, o $p$, em relação à manovacuometria, obteve um valor de $\mathrm{Pi}_{\max } 0,0763 \mathrm{e}$ $\mathrm{Pe}_{\max }$ de 0,4234 , enquanto que no PFE 0 valor de $p$ foi de 0,2161, não apresentando, dessa forma, um teste estatístico com alta significância, porém podem-se visualizar alterações entre os grupos.

É relevante realizar a avaliação do PFE para avaliar a eficiência da função pulmonar e verificar se há ou não colabamento de vias aéreas. Em relação a este, obteve-se diferenças de valores entre os dois grupos estudados, porém, no teste t não foi estatisticamente significativo (tabela 5).

No Grupo 1, se comparado os valores apresentados no teste do PFE, visualiza-se que apresentaram um desempenho maior em comparação ao Grupo 2, ficando em vantagem. Esta diferença não foi de maneira significativa estatisticamente, pois isso pode ocorrer devido à amostra ser pequena; porém, não é um fator limitante, já que se pode notar diferenças quando comparados os dois grupos.

É preciso destacar que os valores do PFE não devem substituir a avaliação clínica, pois é uma medida fortemente influenciada pelo esforço expiratório ${ }^{23}$. Para o manejo da asma, tanto na crian- ça quanto no adulto, a medida do PFE é útil em três aspectos: diagnóstico, avaliação da gravidade e monitoração do tratamento ${ }^{24}$.

Em um estudo foram avaliadas 1.023 crianças na faixa etária de 6 a 12 anos de idade, residentes de uma área urbana da Nigéria, em que se obteve valores médios no PFE de $213,31 / \mathrm{mim}$ e $211 \mathrm{l} /$ mim para meninos e meninas, respectivamente. Os autores também relatam que os valores de PFE encontrados possuem uma correlação significativa com parâmetros antropométricos, principalmente com a estatura ${ }^{25}$.

A mensuração do PFE pode servir para propósitos clínicos e para estudos epidemiológicos, sendo a caracterização da sua variabilidade de grande valia no diagnóstico da hiper-reatividade das vias aéreas e apresenta uma correlação com o escore dos sintomas. Para um monitoramento mais documentado, é monitorado o PFE diariamente, em três momentos (manhã-tarde-noite). Por um tempo de uma semana, é realizada três medidas (escolhendo-se a melhor delas), e é dessa forma que permite identificar e quantificar a variação dos fluxos aéreos nos pacientes portadores de asma brônquica ${ }^{24}$.

As medidas seriadas do PFE auxiliam no diagnóstico de asma quando demonstra-se variabilidade aumentada nos valores obtidos pela manhã e à noite (acima de $20 \%$ em adultos e de $30 \%$ em (rianças) ${ }^{26}$.

Este item da avaliação é de suma importância, pois se sabe que os asmáticos inspiram maior volume de ar do que um indivíduo normal e expiram volume similar, e ao realizar a avaliação do PFE quantifica-se esse volume de ar em litros por minutos. 0 aumento do volume pulmonar no asmático resulta em aumento do diâmetro das vias aéreas, aumentando a contração pulmonar passiva expiratória, compensando o aumento da resistência à expiração, que é maior do que para a inspiração ${ }^{27}$.

\section{Conclusão}

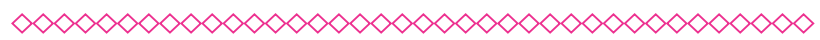

A técnica de mensuração dos valores do PFE, da $\mathrm{Pi}_{\max }$ e de $\mathrm{Pe}_{\text {max }}$ em crianças mostra-se de forma complicada, devido a necessidade da junção entre coordenação e cooperação destas crianças, espe- 
cialmente aquelas situadas na faixa etária abaixo dos 7 anos de idade.

Na análise de dados do PFE, que apresentou no teste $t$ um valor de 0.7958 , pelo simples fato de este ser um valor positivo, já se pode notar que a amostra constituída pelo Grupo 1 obteve valores mais altos, ou seja, apresenta mais força no teste do PFE em comparação ao Grupo 2.

A asma brônquica se trata de uma doença crônica, porém em períodos de crise torna-se agudizada, e é nesse item que enfatizamos que a avaliação das crianças portadoras da patologia não foi realizada no momento das crises, Devido a isso, obtivemos alterações menores, com pouca significância, quando comparada com as crianças sem diagnóstico de asma. Destacamos ainda que não se foi investigado em relação ao Grupo 2 ligações com tratamentos para o controle da asma anteriores que estiveram realizando.

Através do presente estudo, observa-se a importância e a necessidade de se estudar a força muscular respiratória, o fluxo expiratório e a realização de exame físico de tórax em pacientes asmáticos, pois há uma carência de mais estudos para que se estabeleça um consenso a esse respeito, principalmente com crianças, em que verificamos que o número de pesquisas com este público é menor, a fim de produzir maiores conhecimentos a este respeito.

\section{Referências}

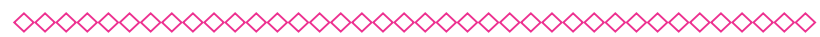

1. Simões SM, Cunha SS, Barreto ML, Cruz AA. Distribuição da gravidade da asma na infância. J. Pediatr. (Rio J.). 2010;86:(5):417-423.

2. Chatkin MN, Menezes AMB, Macedo SEC, Fiss

E. Asma e função pulmonar aos 6-7 anos de idade em uma corte de nascimentos no Sul do Brasil. J. bras. Pneumol. 2008; 34:(10):764-771.

3. Fontes MJF, Fonseca MTM, Camargos PAM, Affonso AGA, Calazans GMC. Asma em menores de cinco anos: dificuldades no diagnóstico e na prescrição da corticoterapia inalatória. J. bras. Pneumol. 2005;31(3):244-253.
4. Ferreira JP. Pediatria: diagnóstico e tratamento. Porto Alegre: Artmed, 2005.

5. Shepherd RB.. Fisioterapia em pediatria. 3. ed. São Paulo Santos, 2002.

6. Sarmento GJV (Org.). Fisioterapia respiratória no paciente crítico: rotinas clínicas. 3. ed. rev. e ampl. São Paulo: Manole, 2010;675.

7. III Consenso Brasileiro no Manejo da Asma (2002). Revista AMRIGS, Porto Alegre, 46 $(3,4): 151-172,2002$.

8. Ribeiro SNS, Fontes MJF, Duarte MA. Avaliação da força muscular respiratória e da função pulmonar por meio de exercício em crianças e adolescentes com asma: ensaio clínico controlado. Pediatria: São Paulo.2010;32(2):98-105.

9. Ezequiel OS. Avaliação e Tratamento do Paciente com Asma Brônquica Revista APS. 2003;6:(1):38-40.

10. Soares E. Metodologia científica: Iógica, epistemologia e normas. São Paulo: Atlas, 2003.

11. Cervo AL, Bervian PA. Metodologia Científica. 5. ed. São Paulo: Pearson Hall, 2002.

12. Azeredo CA. Métodos de avaliação na fisioterapia respiratória. Fisioterapia respiratória moderna. 4 ed. São Paulo: Manole, 2002.

13. Ayres M, Ayres MJ, Ayres DL, Santos AAS. Bioestat: Aplicações estatísticas nas áreas das ciências biomédicas. Belém-PA, 2007.

14. Ferrari FP. Prevalência de asma, rinite alérgica e eczema atópico em escolares de Curitiba-PR [Dissertação de Mestrado]. Curitiba: Universidade Federal do Paraná, 1997.

15. Kamoi TO, Rosário Filho NA, Farias L. História natural da asma em crianças: há remissão na adolescência? Pediatria. 1998;20(4):310-5.

16. Clifford RD, Radford M, Howell JB, Holgate ST. Prevalence of respiratory symptons among 7 and 11 years old schoolchildren and association with asthma. Arch. Dis. Child 1989;64:1118-25. 
17. Junho Sologuren MJ, Solé D, Mallol J, Calil Júnior JA, Freitas AM, Matoso DC. ISAAC (International Study of Asthma and Allergies in Childhood) in one brazilian inner city. J Invest Allergol C, 1995.

18. Guimarães MLLG. Fisioterapia na Asma Brônquica. Pediat. (São Paulo)5:33-37, 1983.

19. Silva APS, Blois LVS, Rosa UA. O Exame Físico do Tórax para o Profissional de Saúde. Santa Fisio, 2000-2010.

20. Rodrigues F, Bárbara C. Pressões Respiratórias Máximas. Proposta de um Protocolo de Procedimentos. Rev Port Pneumol VI, 2000;

(4):297-307.

21. Tobin MJ. Respiratory muscles in disease. Clin. Chest Med, 1988; 9:263-286.

22. Weiner P, Suo J, Fernandez E, Cherniack RM. The efect of hiperinsuflation on respiratory muscle strenght and efficiency in healthy subjects and patients with asthma. Am. Rev. Respire. Dis, 1990;141;1501-1505.
23. Cruz AC. Pico de fluxo expiratório. É melhor medir!. J. Bras Pneumol. 2006; 32

24. Silva LCC, Hetzel, JL. Asma brônquica: manejo clínico. Porto Alegre: Artmed, 1998: 231.

25. Agaba PA, Thacher TD, Angyo IA, Agaba EI. Peak expiratory flow rates in healthy Nigerian children. Journal of Tropical Pediatrics, 2003; 49:(3):157-159.

26. Sociedade Brasileira De Pneumologia E Tisiologia. Diagnóstico clínico e funcional da asma brônquica. Rev. Assoc. Med. Bras. 2004; 50:(2):121-121.

27. Rossi DC, Munhoz DF, Nogueira CR, Oliveira TCM, Britto ATBD. Relação Do Pico De Fluxo Expiratório Com 0 Tempo De Fonação Em Pacientes Asmáticos. Rev CEFAC, São Paulo, 2006; 8:(4):509-17.Nis atur simagna tessum ipicto odis essita aut imolorersped et apidell aborecepra volupta eum estiorpos aciliat.

\section{Anexos}

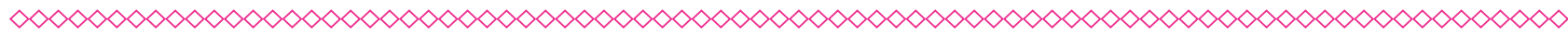

Tabela 1 - Média das idades dos grupos 1 e 2

Médias das idades

\begin{tabular}{lc} 
Grupo 1 & 8,75 \\
Grupo 2 & 8,3 \\
\hline
\end{tabular}

Fonte: elaboração das autoras.

(clique para voltar ao texto)

Tabela 2 - Padrão ventilatório dos grupos 1 e 2

\begin{tabular}{lcc} 
Padrão Ventilatório & Grupo 1 & Grupo 2 \\
Diafragmático & 6 & 3 \\
Intercostal & 6 & 6 \\
Costa Superior & 8 & 11 \\
\hline
\end{tabular}

Fonte: elaboração das autoras. 
Tabela 3 - Tipo de tórax dos grupos 1 e 2

\begin{tabular}{lcc} 
Tipo de tórax & Grupo 1 & Grupo 2 \\
Normal & 8 & 6 \\
Pombo & 2 & 3 \\
Plano & 6 & 4 \\
Tonel & 0 & 3 \\
Sapateiro & 3 & 4 \\
Sino & 1 & 0 \\
\hline
\end{tabular}

Fonte: elaboração das autoras.

Tabela 4 - Média e Desvio Padrão da força muscular respiratória dos grupos 1 e 2

\begin{tabular}{lcccc} 
& \multicolumn{2}{c}{ Grupo 1} & \multicolumn{2}{c}{ Grupo 2 } \\
Manovacuometro & $\mathrm{Pi}_{\text {Max }}$ & $\mathrm{Pe}_{\operatorname{Max}}$ & $\mathrm{Pi}_{\text {Max }}$ & $\mathrm{Pe}_{\operatorname{Max}}$ \\
\hline Média & $64,7 \mathrm{cmH}_{2} \mathrm{O}$ & $65.40 \mathrm{cmH}_{2} \mathrm{O}$ & $78,25 \mathrm{cmH}_{2} \mathrm{O}$ & $63,75 \mathrm{cmH}_{2} \mathrm{O}$ \\
Desvio padrão & 25,67509 & 19,06295 & 31,27599 & 28,23451 \\
\hline
\end{tabular}

$\mathrm{Pe}_{\max }$ : Teste $\mathrm{t}=0.1945, \mathrm{p}=0.4234 / \mathrm{Pi}_{\text {max: }}$ Teste $\mathrm{t}=-1.4596, \mathrm{p}=0.0763$.

Fonte: elaboração das autoras.

(clique para voltar ao texto)

Tabela 5 - Média e Desvio Padrão do pico de fluxo expiratório dos grupos 1 e 2

\begin{tabular}{lcc} 
Peak Flow & Grupo 1 & Grupo 2 \\
Média & $205 \mathrm{I} / \mathrm{min}$ & $186,5 \mathrm{I} / \mathrm{min}$ \\
Desvio padrão & 53,7122 & 85,92293 \\
\hline
\end{tabular}

Teste $\mathrm{t}=0.7958, \mathrm{p}=0.2161$.

Fonte: elaboração das autoras. 